\title{
Correlations of control variables in variational data assimilation
}

\section{Article}

Published Version

Katz, D., Lawless, A. S., Nichols, N. K., Cullen, M. J. P. and Bannister, R. N. (2011) Correlations of control variables in variational data assimilation. Quarterly Journal of the Royal Meteorological Society, 137 (656). pp. 620-630. ISSN 1477870X doi: https://doi.org/10.1002/qj.798 (Part A) Available at https://centaur.reading.ac.uk/19982/

It is advisable to refer to the publisher's version if you intend to cite from the work. See Guidance on citing.

To link to this article DOI: http://dx.doi.org/10.1002/qj.798

Publisher: Royal Meteorological Society

All outputs in CentAUR are protected by Intellectual Property Rights law, including copyright law. Copyright and IPR is retained by the creators or other copyright holders. Terms and conditions for use of this material are defined in the End User Agreement.

\section{www.reading.ac.uk/centaur}

\section{CentAUR}

Central Archive at the University of Reading 
Reading's research outputs online 


\title{
RMetS
}

\section{Correlations of control variables in variational data assimilation}

\author{
D. Katz ${ }^{\mathrm{a}}$, A. S. Lawless ${ }^{\star a}$, N. K. Nichols ${ }^{\mathrm{a}}$, M. J. P. Cullen ${ }^{\mathrm{b} \dagger}$ and R. N. Bannister ${ }^{\mathrm{a}}$ \\ ${ }^{a}$ School of Mathematical and Physical Sciences, University of Reading, UK \\ ${ }^{\mathrm{b}}$ Met Office, Exeter, UK \\ ${ }^{*}$ Correspondence to: A. S. Lawless, Department of Mathematics and Statistics, University of Reading, Whiteknights, \\ PO Box 220, Reading RG6 6AX, Berkshire, UK. E-mail: a.s.lawless@reading.ac.uk \\ ${ }^{\dagger}$ The contribution of this author was written in the course of his employment at the Met Office, UK, and is published with \\ the permission of the Controller of HMSO and the Queen's Printer for Scotland.
}

\begin{abstract}
Variational data assimilation systems for numerical weather prediction rely on a transformation of model variables to a set of control variables that are assumed to be uncorrelated. Most implementations of this transformation are based on the assumption that the balanced part of the flow can be represented by the vorticity. However, this assumption is likely to break down in dynamical regimes characterized by low Burger number. It has recently been proposed that a variable transformation based on potential vorticity should lead to control variables that are uncorrelated over a wider range of regimes. In this paper we test the assumption that a transform based on vorticity and one based on potential vorticity produce an uncorrelated set of control variables. Using a shallow-water model we calculate the correlations between the transformed variables in the different methods. We show that the control variables resulting from a vorticity-based transformation may retain large correlations in some dynamical regimes, whereas a potential vorticitybased transformation successfully produces a set of uncorrelated control variables. Calculations of spatial correlations show that the benefit of the potential vorticity transformation is linked to its ability to capture more accurately the balanced component of the flow. Copyright (c) 2011 Royal Meteorological Society and British Crown Copyright, the Met Office
\end{abstract}

\begin{abstract}
Key Words: Burger number; control variable transform; potential vorticity; shallow-water model
Received 13 April 2010; Revised 24 January 2011; Accepted 28 January 2011; Published online in Wiley Online Library 16 March 2011
\end{abstract}

Citation: Katz D, Lawless AS, Nichols NK, Cullen MJP, Bannister RN. 2011. Correlations of control variables in variational data assimilation. Q. J. R. Meteorol. Soc. 137: 620-630. DOI:10.1002/qj.798

\section{Introduction}

An important component of three- and four-dimensional variational data assimilation schemes is the backgrounderror covariance matrix. This matrix, which specifies the covariances of the a priori forecast errors, determines the way in which information from observed variables is spread to unobserved variables and to unobserved spatial points (Bannister, 2008a). For operational weather forecasting, the huge size of the system state means that a full representation of this matrix is impossible and so the matrix is constructed implicitly by means of a variable transformation. The analysis is generally performed with a set of variables different from the model variables, known as control variables, and a transformation of these control variables to the original model variables is defined (Lorenc et al., 2000; Bannister, 2008b). An important assumption made in building a variational data assimilation system in this way is that the forecast errors in the control variables chosen are statistically independent, so that no cross-covariance information needs to be defined in the space of control variables.

In practice the variable transformation from model to control variables consists of several stages. The first stage is a 
transformation to a set of physical variables whose errors are assumed to be uncorrelated between themselves, but which may contain spatial correlations. This part is known as the parameter transform. Further transformations are then used to remove spatial correlations in the horizontal and vertical directions (Bannister, 2008b). A natural way of performing the parameter transform is to partition the flow into balanced and unbalanced components. For the linearized system, these components are linearly independent and so there is no dynamical interaction between them. Thus we may expect that in a weakly nonlinear regime errors in control variables based on such a partitioning will be largely uncorrelated. For the atmosphere, it is known that the balanced component of the flow is well characterized by the advection and inversion of potential vorticity (PV) and so a parameter transform based on PV would be expected to give a good decomposition of the flow variables (Hoskins et al., 1985). In practice for certain dynamical regimes, characterized by high Burger number, the PV is well approximated by the vorticity. In this case the inversion reduces from a three-dimensional to a two-dimensional problem that is much easier to solve. Thus Parrish and Derber (1992) proposed a parameter transform based on the vorticity rather than the PV. In that paper, and in many operational data assimilation systems since, it is assumed that the rotational part of the flow is completely balanced and so the balance is completely captured by the vorticity (Derber and Bouttier, 1999; Gauthier et al., 1999; Lorenc et al., 2000; Lorenc, 2003; Barker et al., 2004; Fischer et al., 2005; Zupanski et al., 2005; Bannister, 2008b). While this is true for many flow regimes, the approximation is likely to break down on scales larger than the Rossby radius of deformation. In operational systems, the lack of validity in these dynamical regimes is usually accounted for by a statistical regression technique. However, this results in a univariate analysis in these regimes and the true correlations between the mass and wind variables are ignored.

It is expected that these problems could be eliminated by the use of a parameter transformation based on PV rather than vorticity as the balanced variable. Wlasak et al. (2006) showed, in the context of a two-dimensional shallow-water model, that a PV-based control variable could capture the true balance of the system over a wider range of regimes than a vorticity-based control variable. In the context of operational models, Cullen (2003) and Bannister and Cullen (2007) performed initial studies with a PV-based control variable transform in the models of the European Centre for Medium-range Weather Forecasting (ECMWF) and the Met Office respectively. However, the elliptic equations in the transforms for the ECMWF and Met Office operational systems were ill-conditioned, which made them difficult to solve accurately. This problem was made worse in the ECMWF system by the Lorenz vertical grid staggering, which led to the need for extra regularization to make the problem well-posed.

In this article, we extend these previous studies by testing the fundamental assumption that the errors in the balanced and unbalanced control variables are uncorrelated when the vorticity-based and PV-based parameter transforms are used. The need for numerical compromises in the studies of Cullen (2003) and Bannister and Cullen (2007) meant that a clean comparison between the two transforms was not possible. Here we use a shallow-water model to demonstrate the fundamental differences between the two transforms numerically and to compare these numerical results to theory. We use the shallow-water model to calculate correlation statistics between the different control variables to test how well the transforms remove correlations by splitting the flow into its balanced and unbalanced parts. We show that, for certain dynamical regimes, the assumption of zero correlations between control variables is valid when the PV-based transform is used, but not with the vorticity-based transform. These results give further details of the findings presented briefly in Bannister et al. (2008). Furthermore, we calculate the spatial correlations for each control variable and use these to understand the effectiveness of the parameter transforms at decoupling the balanced and unbalanced parts of the flow.

The outline of the remainder of the article is as follows. In section 2 we present the model used in this study, in its continuous and discrete forms. In section 3 we present the two different parameter transforms as applied to this model. Section 4 examines briefly the covariance structures implied by these transforms. In section 5 we present the statistics of the correlations between control variables in different dynamical regimes. Section 6 presents the correlation functions of the different control variables, which allow us to diagnose how well the balanced part of the flow is captured in the transforms. Finally we summarize the results in section 7 .

\section{The model}

\subsection{Continuous system}

The model we use for this study is the one-dimensional shallow-water equations with rotation and orography, in the presence of a constant mean flow. The model equations are given by

$$
\begin{aligned}
\frac{\mathrm{D} u}{\mathrm{D} t}+\frac{\partial \phi}{\partial x}+g \frac{\partial \widetilde{H}}{\partial x}-f v & =0, \\
\frac{\mathrm{D} v}{\mathrm{D} t}+f u & =0, \\
\frac{\mathrm{D} \ln \phi}{\mathrm{D} t}+\frac{\partial u}{\partial x} & =0,
\end{aligned}
$$

where

$$
\frac{\mathrm{D}}{\mathrm{D} t} \equiv \frac{\partial}{\partial t}+\left(U_{\mathrm{c}}+u\right) \frac{\partial}{\partial x}
$$

and $\phi=g h$. In these equations, $u$ denotes the departure of the velocity in the $x$-direction from a known constant forcing mean flow $U_{c}, v$ is the velocity in the $y$-direction, $h$ is the height of the fluid, $\phi$ is the geopotential, $\widetilde{H}=\widetilde{H}(x)$ is the height of the orography, $f$ is the constant Coriolis parameter and $g$ is the gravitational force. The model assumes that there is no variation in the $y$-direction and the boundary conditions in the $x$-direction are taken to be periodic, with $x \in[0, l]$.

This model is chosen as it is the simplest system that contains key properties required to define the vorticity-based and PV-based parameter transforms. In particular, we have a non-trivial first order geostrophic balance relationship

$$
f v=g \frac{\partial(h+\widetilde{H})}{\partial x} .
$$


This relationship is found through an asymptotic expansion in small Rossby number (Pedlosky, 1987), where the Rossby number is defined as the dimensionless parameter

$$
R o=\frac{U}{f L},
$$

and $U$ and $L$ are characteristic velocity- and length-scales respectively. At small Rossby number, the balance (5) is dominant at horizontal length-scales that are larger than the Rossby radius of deformation $L_{\mathrm{r}}$, defined as

$$
L_{\mathrm{r}}=\frac{\sqrt{g H}}{f},
$$

where $H$ is a characteristic depth-scale. The shallow-water equations also conserve the PV, defined by

$$
q=\frac{1}{h}\left(f+\frac{\partial v}{\partial x}\right) .
$$

In order to characterize the differing flow regimes in this system, we will make use of the Burger number, which is defined as

$$
B u=\frac{\sqrt{g H}}{f L}
$$

(Wlasak et al., 2006). The Burger number is a measure of the relative importance of rotation and stratification in the flow. It is the ratio of the Rossby number and the Froude number

$$
F r=\frac{U}{\sqrt{g H}} .
$$

The Froude number is the ratio of the advective velocity to the gravity wave speed. In most deep atmospheric motions, $\mathrm{Fr}$ is small, that is the advective velocity is much less than the gravity wave speed. The two components on the right-hand side of the PV equation (8) take on a different importance as the Burger number changes. For small Burger number regimes, the $\mathrm{PV}$ is dominated by the first term, $f / h$, whereas in regimes of high Burger number the PV can be well approximated by $(\partial v / \partial x) / h$ (Wlasak et al., 2006).

\subsection{Discrete model}

The model (1) to (3) is discretized using a semi-implicit, semi-Lagrangian scheme, following a similar scheme to Lawless et al. (2003). This gives the following time-discrete equations

$$
\begin{aligned}
& \frac{u_{\mathrm{a}}^{n+1}-u_{\mathrm{d}}^{n}}{\Delta t}+\alpha_{1}\left[\phi_{x}+g \widetilde{H}_{x}-f v\right]_{\mathrm{a}}^{n+1} \\
&+\left(1-\alpha_{1}\right)\left[\phi_{x}+g \widetilde{H}_{x}-f v\right]_{\mathrm{d}}^{n}=0, \\
& \frac{v_{\mathrm{a}}^{n+1}-v_{\mathrm{d}}^{n}}{\Delta t}+\alpha_{2}[f u]_{\mathrm{a}}^{n+1}+\left(1-\alpha_{2}\right)[f u]_{\mathrm{d}}^{n}=0, \\
& \frac{\ln \phi_{\mathrm{a}}^{n+1}-\ln \phi_{\mathrm{d}}^{n}}{\Delta t}+\alpha_{3}\left[u_{x}\right]_{\mathrm{a}}^{n+1}+\left(1-\alpha_{3}\right)\left[u_{x}\right]_{\mathrm{d}}^{n}=0,
\end{aligned}
$$

where the subscript $x$ denotes the derivative with respect to $x$, subscripts a and $\mathrm{d}$ denote arrival and departure points respectively, and superscripts denote the time level. The discrete equations are solved on a grid where the velocities $u$ and $v$ are held on points staggered from those where the height $h$ is held, that is, on a one-dimensional analogue of the Arakawa $\mathrm{B}$ grid. The time-weighting parameters $\alpha_{1}, \alpha_{2}$ and $\alpha_{3}$ are chosen to meet the stability requirements of the scheme. Further details about the discrete solution of the differential equations can be found in Katz (2007).

\section{Parameter transforms}

The transformation from model variables to control variables is used within the incremental formulation of variational data assimilation. In this formulation of the assimilation problem, the minimization of the nonlinear variational cost function is replaced by a series of minimizations of linear least-squares cost functions (Courtier et al., 1994; Lorenc et al., 2000). We let $\mathbf{x}$ represent the model state. In incremental variational data assimilation, the nonlinear cost function is linearized about the current state estimate $\overline{\mathbf{x}}$ and this linear least-squares cost function is minimized for an increment $\mathbf{x}^{\prime}$ in what is known as the 'inner loop' minimization. The increment can then be added to the estimate $\overline{\mathbf{x}}$ in an 'outer loop' step to provide a better estimate of the system state and the process repeated as required. This is equivalent to applying an approximate Gauss-Newton iteration to solve the nonlinear minimization problem (Lawless et al., 2005).

The parameter transform occurs within the definition of the inner loop minimization problem. We define a control variable $\mathbf{z}^{\prime}$ and a transformation $\mathbf{U}$ from control variables to model variables

$$
\mathbf{x}^{\prime}=\mathbf{U z}^{\prime}
$$

(the U-transform). In practice, the control variable vector might not be the same length as the vector of original model variables, and so the matrix $\mathbf{U}$ is not necessarily square (Lorenc et al., 2000). The inverse transform from model to control variables is defined by

$$
\mathbf{z}^{\prime}=\mathbf{T} \mathbf{x}^{\prime}
$$

(the T-transform), where $\mathbf{T}$ is a generalized inverse of $\mathbf{U}$. The inner loop minimization problem is then defined in terms of the control variables $\mathbf{z}^{\prime}$, which are assumed to be independent. The solution to the minimization problem can then be mapped to the space of model variables using the U-transform in order to update the state estimate in the outer loop step.

We note here that normally the transformation $\mathbf{U}$ is defined to include also the transformation of the spatial covariances to spectral space (e.g. Lorenc et al., 2000). Here we use the notation $\mathbf{U}$ to imply only the parameter transform. Hence the background-error covariance matrix $\mathbf{B}$ in the space of model variables is given by

$$
\mathbf{B}=\mathbf{U} \boldsymbol{\Lambda} \mathbf{U}^{\mathrm{T}},
$$

where $\boldsymbol{\Lambda}$ is a block-diagonal matrix with the blocks corresponding to auto-correlations for each of the control variables. We now set out in detail the vorticity-based and PV-based parameter transforms for the one-dimensional shallow-water model introduced in the previous section. 
For this system, the linearization state $\overline{\mathbf{x}}$ and the increment field $\mathbf{x}^{\prime}$ are defined by the vectors

$$
\overline{\mathbf{x}}=\left(\begin{array}{c}
\bar{u} \\
\bar{v} \\
\bar{h}
\end{array}\right), \quad \mathbf{x}^{\prime}=\left(\begin{array}{c}
u^{\prime} \\
v^{\prime} \\
h^{\prime}
\end{array}\right) .
$$

In order to decompose the shallow-water flow into its balanced and unbalanced components, we perform a normal mode analysis of equations (1)-(3) in the absence of a mean flow $U_{c}$, linearized around a state of rest. Such an analysis reveals the presence of three normal modes, one slow mode and two fast modes. The slow mode is taken to be the balanced part of the flow and satisfies the linearization of the PV equation (8)

$$
q^{\prime} \bar{h}=\frac{\partial v^{\prime}}{\partial x}-\bar{q} h^{\prime}
$$

where $\bar{q}$ is the linearization state of the PV. The corresponding first order linear balance equation is given by a linearization of (5), which leads to

$$
f v^{\prime}-g \frac{\partial h^{\prime}}{\partial x}=0
$$

The two remaining modes represent the unbalanced part of the flow and can be related to the departure from balance and to the divergence. A decomposition of flow into balanced and unbalanced variables has previously been used to develop numerical methods for solving the flow equations (Dritschel et al., 1999; Mohebalhojeh and Dritschel, 2004). Here we use such a decomposition to design the parameter transforms for incremental variational data assimilation. We seek three control variables for the data assimilation problem which represent one balanced and two unbalanced components of the flow.

In order to derive both the vorticity-based and PVbased transforms for our model, we make use of Wlasak et al. (2006), who derived the corresponding T-transforms for the two-dimensional shallow-water equations on the sphere, and Cullen (2003), who derived the PV-based transform for an operational numerical weather prediction model. Appropriate transforms are defined for the onedimensional shallow-water model used in this study. We first consider a separation of the flow into its rotational and divergent parts by means of a Helmholtz decomposition. We define a stream function $\psi^{\prime}$ and a velocity potential $\chi^{\prime}$. Then for the one-dimensional shallow-water model, the Helmholtz decomposition reduces to the vorticity

$$
\zeta^{\prime}=\frac{\partial v^{\prime}}{\partial x}=\frac{\partial^{2} \psi^{\prime}}{\partial x^{2}}
$$

and the divergence

$$
\mathcal{D}^{\prime}=\frac{\partial u^{\prime}}{\partial x}=\frac{\partial^{2} \chi^{\prime}}{\partial x^{2}}
$$

with velocities $u^{\prime}$ and $v^{\prime}$ given by

$$
\begin{aligned}
& u^{\prime}=\frac{\partial \chi^{\prime}}{\partial x}, \\
& v^{\prime}=\frac{\partial \psi^{\prime}}{\partial x} .
\end{aligned}
$$

\subsection{Vorticity-based transform}

The vorticity-based transform makes the assumption that the rotational component of the wind is totally balanced. We define a balanced height variable $h_{\mathrm{b}}^{\prime}$ which is related to the stream function through the linear balance equation (19). Thus we obtain

$$
f \frac{\partial^{2} \psi^{\prime}}{\partial x^{2}}-g \frac{\partial^{2} h_{\mathrm{b}}^{\prime}}{\partial x^{2}}=0 .
$$

For the case of periodic boundary conditions, this can be integrated to obtain

$$
h_{\mathrm{b}}^{\prime}=\frac{f}{g} \psi^{\prime} .
$$

The control variables are then taken to be the stream function, the velocity potential and the unbalanced (residual) component of the height field, defined by

$$
h_{\mathrm{res}}^{\prime}=h^{\prime}-h_{\mathrm{b}}^{\prime} \text {. }
$$

In operational systems, this unbalanced height component is represented by an unbalanced pressure (Lorenc et al., 2000). The T-transform, from model variables $u^{\prime}, v^{\prime}, h^{\prime}$ to control variables $\chi^{\prime}, h_{\text {res }}^{\prime}, \psi^{\prime}$, then proceeds as described for the two-dimensional shallow-water equations by Wlasak et al. (2006) with the following steps:

1. Find the velocity potential $\chi^{\prime}$ from $u^{\prime}$ using (21);

2. Find the stream function $\psi^{\prime}$ from $v^{\prime}$ using (20);

3. Calculate the balanced height $h_{\mathrm{b}}^{\prime}$ using (25);

4. Calculate the residual height $h_{\text {res }}^{\prime}$ using (26).

The solutions of (21) and (20) are unique up to a constant, which is chosen to ensure that the mean values of $\psi^{\prime}$ and $\chi^{\prime}$ are zero. By choosing the constant in this way, we lose a degree of freedom in each equation. These missing degrees of freedom are used to retain the mean values of the wind components that are lost through differentiation. Thus the mean values, which we denote $\left\langle u^{\prime}\right\rangle$ and $\left\langle v^{\prime}\right\rangle$, are also control variables.

The U-transform, from control variables to model variables, is defined in the following way:

1. Calculate the velocity $v^{\prime}$ from $\psi^{\prime}$ and $<v^{\prime}>$ :

$$
v^{\prime}=\frac{\partial \psi^{\prime}}{\partial x}+<v^{\prime}>
$$

2. Calculate the balanced height increment $h_{\mathrm{b}}^{\prime}$ from $\psi^{\prime}$ using (25);

3. Calculate the full height increment $h^{\prime}$ from $h_{\mathrm{b}}^{\prime}$ using (26);

4. Calculate the velocity $u^{\prime}$ from $\chi^{\prime}$ and $<u^{\prime}>$ :

$$
u^{\prime}=\frac{\partial \chi^{\prime}}{\partial x}+<u^{\prime}>
$$

It is useful to note that the consideration of the mean values is more natural in the implementation of the transforms in operational systems such as that of the Met Office, where the transforms are solved in spectral space (Lorenc et al., 2000). For these systems, the transform is only applied to wave numbers one and above, and wave number zero, which holds the mean values, is not transformed. It is the lack of a spectral transform in our study that makes necessary a special treatment of the mean values. 


\subsection{PV-based transform}

For the PV-based transform, we allow the stream function to have both balanced and unbalanced components, which we denote $\psi_{\mathrm{b}}^{\prime}$ and $\psi_{\mathrm{u}}^{\prime}$ respectively, with corresponding balanced and unbalanced winds $v_{\mathrm{b}}^{\prime}$ and $v_{\mathrm{u}}^{\prime}$ defined by the Helmholtz decomposition. In a similar way, the height is split into balanced and unbalanced components $h_{\mathrm{b}}$ and $h_{\mathrm{u}}$. We assume that the linearized PV is associated solely with the balanced variables and that the balanced variables satisfy the linear balance equation, with the unbalanced variables satisfying departure from this balance. Thus from (18) and (19) we obtain

$$
\begin{aligned}
f \frac{\partial^{2} \psi_{\mathrm{b}}^{\prime}}{\partial x^{2}}-g \frac{\partial^{2} h_{\mathrm{b}}^{\prime}}{\partial x^{2}} & =0, \\
\frac{\partial^{2} \psi_{\mathrm{b}}^{\prime}}{\partial x^{2}}-\bar{q} h_{\mathrm{b}}^{\prime} & =q^{\prime} \bar{h}, \\
f \frac{\partial^{2} \psi_{\mathrm{u}}^{\prime}}{\partial x^{2}}-g \frac{\partial^{2} h_{\mathrm{u}}^{\prime}}{\partial x^{2}} & =\zeta_{\mathrm{a}}^{\prime}, \\
\frac{\partial^{2} \psi_{\mathrm{u}}^{\prime}}{\partial x^{2}}-\bar{q} h_{\mathrm{u}}^{\prime} & =0,
\end{aligned}
$$

where $\zeta_{a}^{\prime}$, the departure from geostrophic balance, is defined by the equation

$$
\zeta_{a}^{\prime}=f \frac{\partial^{2} \psi^{\prime}}{\partial x^{2}}-g \frac{\partial^{2} h^{\prime}}{\partial x^{2}}
$$

The control variables are then taken to be the balanced stream function $\psi_{\mathrm{b}}^{\prime}$, the unbalanced height $h_{\mathrm{u}}^{\prime}$ and the velocity potential $\chi^{\prime}$. We note that other combinations of balanced and unbalanced variables could theoretically be chosen, but this set is the easiest to implement numerically and is the most well conditioned (Cullen, 2003; Wlasak et al., 2006).

Integration of (29) with periodic boundary conditions gives

$$
h_{\mathrm{b}}^{\prime}=\frac{f}{g} \psi_{\mathrm{b}}^{\prime} .
$$

This can be substituted into (30) to obtain a second-order equation for $\psi_{\mathrm{b}}^{\prime}$,

$$
\frac{\partial^{2} \psi_{\mathrm{b}}^{\prime}}{\partial x^{2}}-\frac{f \bar{q}}{g} \psi_{\mathrm{b}}^{\prime}=q^{\prime} \bar{h},
$$

which is the PV inversion equation for this system. The Ttransform, from model variables $u^{\prime}, v^{\prime}, h^{\prime}$ to control variables $\chi^{\prime}, h_{\mathrm{u}}^{\prime}, \psi_{\mathrm{b}}^{\prime}$, follows Wlasak et al. (2006) and is given by the following steps:

1. Find the velocity potential $\chi^{\prime}$ from $u^{\prime}$ using (21);

2. Solve (35) for $\psi_{\mathrm{b}}^{\prime}$;

3. Evaluate $h_{\mathrm{b}}^{\prime}$ using (34) and set $h_{\mathrm{u}}^{\prime}=h^{\prime}-h_{\mathrm{b}}^{\prime}$;

4. Store the mean values of $u^{\prime}$ and $v^{\prime}$ which are lost through differentiation.

The U-transform, from control variables to model variables, is defined as follows:

1. Calculate the balanced velocity increment $v_{\mathrm{b}}^{\prime}$ from $\psi_{\mathrm{b}}^{\prime}$ using (23);
2. Calculate $\psi_{\mathrm{u}}^{\prime}$ from $h_{\mathrm{u}}^{\prime}$ using (32) and the unbalanced velocity increment from $\psi_{\mathrm{u}}^{\prime}$ using (23) and reconstruct the full velocity increment $v^{\prime}$ :

$$
v^{\prime}=v_{\mathrm{b}}^{\prime}+v_{\mathrm{u}}^{\prime}+<v>
$$

3. Calculate the balanced height increment $h_{\mathrm{b}}^{\prime}$ from $\psi_{\mathrm{b}}^{\prime}$ using (34) and reconstruct the full height increment $h^{\prime}=h_{\mathrm{b}}^{\prime}+h_{\mathrm{u}}^{\prime}$;

4. Calculate the velocity $u^{\prime}$ from $\chi^{\prime}$ and $<u>$ :

$$
u^{\prime}=\frac{\partial \chi^{\prime}}{\partial x}+<u>
$$

The derivation of these transforms assumes that the equation for the unbalanced stream function (32) with periodic boundary conditions has a solution. This will be true only if the mean value of $\bar{q} h_{\mathrm{u}}^{\prime}$ is zero. Within the data assimilation system, this condition may not be satisfied unless it is explicitly enforced. It is not straightforward to understand how to do this, since $\bar{q}$ varies with $x$ and is modified on every outer loop of the incremental variational data assimilation. It is possible to modify $h_{\mathrm{u}}^{\prime}$ on each inner iteration so that the mean of $\bar{q} h_{\mathrm{u}}^{\prime}$ is zero. This can be achieved since we are always able to subtract a constant from $h_{\mathrm{u}}^{\prime}$ such that $<\bar{q} h_{\mathrm{u}}^{\prime}>=0$. The mean of the full height increment is therefore split between $h_{\mathrm{b}}^{\prime}$ and $h_{\mathrm{u}}^{\prime}$.

The problem could be avoided by choosing to approximate $\bar{q}$ by a constant. An approximation of this sort was made in Cullen (2003). This would mean that we are simply able to set $\left\langle h_{\mathrm{u}}^{\prime}\right\rangle=0$ and the condition $<\bar{q} h_{\mathrm{u}}^{\prime}>=0$ will always be satisfied. We then store the mean of the full height increment solely in $h_{\mathrm{b}}^{\prime}$. This approximation may be desirable from an operational perspective since the transform is then less computationally demanding. In section 5 we consider the possible implications of making this approximation in the PV-based transform. We choose to make the approximation

$$
\bar{q}=\frac{1}{\bar{h}}\left(f+\frac{\partial \bar{v}}{\partial x}\right) \approx \frac{f}{<\bar{h}>},
$$

which essentially assumes that $\partial \bar{v} / \partial x=0$. This is consistent with the climatological assumptions and zonally averaged quantities used in determining the background-error covariance matrix in practice. However, the use of this approximation would weaken the dependence of the assumed background errors on the current state of the flow, since the state only appears as a constant spatial average of $h$, which is updated on each outer loop iteration.

Having presented both the vorticity-based and PV-based parameter transforms for the one-dimensional shallowwater model, we now consider the covariance structure implied by these transforms.

\section{Implied covariance structures}

We can understand something of the difference between the two parameter transforms presented by looking at the implied background-error covariance matrix by means of (16). For ease of presentation, we do not consider the mean values of the increments in this analysis, although they are included in the numerical calculations discussed in the remainder of this article. 
For the vorticity-based transform, the control variables are $\chi^{\prime}, \psi^{\prime}$ and $h_{\text {res }}^{\prime}$ and the discrete form of the U-transform defined in section 3.1 can be written in matrix notation as

$$
\left(\begin{array}{c}
u^{\prime} \\
v^{\prime} \\
h^{\prime}
\end{array}\right)=\left(\begin{array}{ccc}
\Delta_{x} & 0 & 0 \\
0 & \Delta_{x} & 0 \\
0 & f / g & 1
\end{array}\right)\left(\begin{array}{c}
\chi^{\prime} \\
\psi^{\prime} \\
h_{\mathrm{res}}^{\prime}
\end{array}\right),
$$

where $\Delta_{x}$ is the matrix representing the discretization of the first-derivative operator $\partial / \partial x$ on the periodic domain $[0, l]$. Therefore the background-error covariance matrix for model variables $u^{\prime}, v^{\prime}$ and $h^{\prime}$ implied by the transform is

$$
\mathbf{B}_{\mathrm{V}}=\left(\begin{array}{ccc}
\Delta_{x} \boldsymbol{\Lambda}_{\chi} \Delta_{x}^{\mathrm{T}} & 0 & 0 \\
0 & \Delta_{x} \boldsymbol{\Lambda}_{\psi} \Delta_{x}^{\mathrm{T}} & \Delta_{x} \boldsymbol{\Lambda}_{\psi} \frac{f}{g} \\
0 & \left(\Delta_{x} \boldsymbol{\Lambda}_{\psi} \frac{f}{g}\right)^{\mathrm{T}} & \left(\frac{f}{g}\right)^{2} \boldsymbol{\Lambda}_{\psi}+\boldsymbol{\Lambda}_{h_{\mathrm{r}}}
\end{array}\right),
$$

where $\boldsymbol{\Lambda}_{\chi}, \boldsymbol{\Lambda}_{\psi}$ and $\boldsymbol{\Lambda}_{h_{\mathrm{r}}}$ are the autocovariance matrices for $\chi^{\prime}, \psi^{\prime}$ and $h_{\text {res }}^{\prime}$ respectively and form the blocks of the block-diagonal matrix $\boldsymbol{\Lambda}$ defined in (16).

For the PV-based parameter transform defined in section 3.2, the control variables are $\chi^{\prime}, \psi_{\mathrm{b}}^{\prime}$ and $h_{\mathrm{u}}^{\prime}$. For this case the discrete $\mathrm{U}$-transform can be written in matrix notation as

$$
\left(\begin{array}{c}
u^{\prime} \\
v^{\prime} \\
h^{\prime}
\end{array}\right)=\left(\begin{array}{ccc}
\Delta_{x} & 0 & 0 \\
0 & \Delta_{x} & \mathbf{Q} \\
0 & f / g & 1
\end{array}\right)\left(\begin{array}{c}
\chi^{\prime} \\
\psi_{\mathrm{b}}^{\prime} \\
h_{\mathrm{u}}^{\prime}
\end{array}\right),
$$

where the matrix $\mathbf{Q}$ is the discrete operator such that

$$
\mathbf{Q} h_{\mathrm{u}}^{\prime} \approx \frac{\partial}{\partial x}\left(\nabla^{-2} \bar{q} h_{\mathrm{u}}^{\prime}\right)
$$

on the periodic domain $[0, l]$, with $\nabla^{2} \equiv \partial^{2} / \partial x^{2}$. The implied background-error covariance matrix is then

$$
\begin{aligned}
& \mathbf{B}_{\mathrm{PV}}= \\
& \left(\begin{array}{ccc}
\Delta_{x} \boldsymbol{\Lambda}_{\chi} \Delta_{x}^{\mathrm{T}} & 0 & 0 \\
0 & \Delta_{x} \boldsymbol{\Lambda}_{\psi_{\mathrm{b}}} \Delta_{x}^{\mathrm{T}}+\mathbf{Q} \boldsymbol{\Lambda}_{h_{\mathrm{u}}} \mathbf{Q}^{\mathrm{T}} & \Delta_{x} \boldsymbol{\Lambda}_{\psi_{\mathrm{b}}} \frac{f}{g}+\mathbf{Q} \boldsymbol{\Lambda}_{h_{\mathrm{u}}} \\
0 & \left(\Delta_{x} \boldsymbol{\Lambda}_{\psi_{\mathrm{b}}} \frac{f}{g}+\mathbf{Q} \boldsymbol{\Lambda}_{h_{\mathrm{u}}}\right)^{\mathrm{T}} & \left(\frac{f}{g}\right)^{2} \boldsymbol{\Lambda}_{\psi_{\mathrm{b}}}+\boldsymbol{\Lambda}_{h_{\mathrm{u}}}
\end{array}\right)
\end{aligned}
$$

where $\boldsymbol{\Lambda}_{\chi}, \boldsymbol{\Lambda}_{\psi_{\mathrm{b}}}$ and $\boldsymbol{\Lambda}_{h_{\mathrm{u}}}$ are the autocovariance matrices for $\chi^{\prime}, \psi_{\mathrm{b}}^{\prime}$ and $h_{\mathrm{u}}^{\prime}$ respectively and form the blocks of the blockdiagonal matrix $\boldsymbol{\Lambda}$ defined in (16) for the PV-transform.

We note first that for both $\mathbf{B}_{\mathrm{V}}$ and $\mathbf{B}_{\mathrm{PV}}$ there are no implied covariances between $u^{\prime}$ and other model variables. This de-coupling of $u$ is a result of assuming that there is no variation in the $y$ direction in the one-dimensional shallowwater model and hence the divergence depends solely on $u$. This would not be true in a more general model. Implied covariance with $u$ could be introduced into this model by splitting the $u$ into balanced and unbalanced components We would then need a relationship defining a balanced component of $u$ (e.g. Fletcher, 2004; Wlasak et al., 2006).

A comparison of $\mathbf{B}_{\mathrm{V}}$ and $\mathbf{B}_{\mathrm{PV}}$ shows that the differences between the implied background-error statistics occur in the auto-correlations of the $v$ field and in the cross-correlations between $v$ and $h$. For each of these, we see that the PVbased implied background-error statistics depend on the currrent dynamical state through the dependence on $\bar{q}(x, t)$, the linearization state of the PV. The state dependence introduces a mechanism to change the implied background statistics on each outer iteration of the incremental 4D-Var algorithm through the linearisation state. In the case where the approximate PV (38) is used in the transform, the state dependence arises only through the current mean value of the $\bar{h}$ field, which is updated on each outer loop. The statistics implied by the vorticity-based transform on the other hand have no state dependence and so are not updated when using this transform. Thus other mechanisms must be used if we wish to introduce state-dependent background statistics when using the vorticity-based transform. For example, some state dependence is also included in the transform used at ECMWF, where the linear balance equation in the vorticity transform is replaced by a nonlinear balance operator, which is then linearized for use in the incremental 4D-Var system (Fisher, 2003).

\section{Correlations between control variables}

Having presented an analysis of the two different parameter transforms, we examine how well the transforms remove correlations between control variables. In particular, we wish to understand whether the balanced and unbalanced components of the flow, as described by the transform, really are uncorrelated; whether the PV-based transform is more successful at removing correlations between variables; and what the consequences are of using the approximate linearization state PV (38) in the transform. In order to address these questions, we generate statistics of the correlations between background errors as represented by the different control variables. To do this we use the numerical model introduced in section 2.2. First we discuss the methodology we use to generate the correlation statistics.

\subsection{Methodology}

A common method for generating background-error covariance statistics in data assimilation is that introduced by Parrish and Derber (1992) and referred to as the 'NMC method'. This method uses differences between forecasts of different lead times for the same validity time as a proxy for forecast errors. A large sample of such differences is created, over which statistics can be calculated. A disadvantage with this method is that it requires an existing data assimilation system before the statistics can be calculated.

An alternative approach, presented by Polavarapu et al. (2005), is to generate one long integration of the numerical model and take differences between the forecast fields at constant intervals of time apart as a proxy for forecast errors. Statistics can then be taken over a sample of these forecast differences. It is this method which we use to generate the correlation statistics in this article. The correlations between different control variables are calculated by first transforming the forecast differences using the appropriate T-transform to give a set of increments in the space of control variables and then using these increments as a statistical sample. The linearization state $\overline{\mathbf{x}}$ used in the transform is given by the full model field at the start of each time interval. In the calculation of the statistics, the values of the forecast differences at each grid point and for each time interval are assumed to be statistically independent and so the total sample size is the number of grid points multiplied by the number of time intervals. We note that one characteristic of the approach of Polavarapu et al. (2005) is that the largest forecast differences are always associated with the highest frequencies (Jackson et al., 2008).

For the experiments presented in this paper, we discretize the model using 500 grid points and take 100 time differences, giving a total statistical sample of size 50000 . This sample size was chosen as that for which the correlation statistics were seen to have converged in a series of experiments with different sample sizes. The model grid 
spacing is taken to be $\Delta x=12.5 \mathrm{~m}$, which means that the domain length $l=6250 \mathrm{~m}$, the model time step $\Delta t=2.5 \mathrm{~s}$, the Coriolis parameter $f=0.01 \mathrm{~s}^{-1}$ and the time-weighting parameters $\alpha_{1}, \alpha_{2}, \alpha_{3}$ are set to 0.6 . The model orography is defined by

$$
\widetilde{H}(x)=H_{\mathrm{c}}\left(1-\frac{x^{2}}{a^{2}}\right),
$$

where $a=40 \Delta x$ and $H_{c}$ is a mean height. The model is run in two different dynamical regimes, defined by high and low Burger number. For the high Burger regime, we set $H_{\mathrm{c}}=7.6 \mathrm{~m}$ and for the low Burger regime we have $H_{\mathrm{c}}=0.019 \mathrm{~m}$. With these parameters, the Burger numbers of the different regimes are 4.0 and 0.2 . The initial conditions for the model are defined to be a state of rest, with $h$ a constant height above the orography of $40 \mathrm{~m}$ in the high Burger regime and $0.1 \mathrm{~m}$ in the low Burger regime. These initial conditions are in geostrophic balance, but unbalanced motions are then created by the presence of the orography. This ensures that there is a mixture of balanced and unbalanced modes in the solution fields, which thus provides a strong test for the parameter transforms. Different experiments are performed in which the mean flow $U_{\mathrm{c}}$ is varied from 0.1 to $5.0 \mathrm{~m} \mathrm{~s}^{-1}$, which varies the Rossby number between 0.02 and 1.0.

In order to generate the statistics, we must choose an appropriate time interval for the forecast differences. When the NMC method is used to generate statistics for operational systems, the forecasts are taken to be $24 \mathrm{~h}$ apart, so as to remove the diurnal cycle, which would otherwise dominate the statistics. Polavarapu et al. (2005) use a $6 \mathrm{~h}$ interval in their method, but the time differences are then adjusted to account for diurnal changes. In a similar way, for the shallow-water model used here, we must choose the time interval over which we difference the model fields in order to remove any similar dominant signal which may be present. To identify the dominant signal, we generate a forecast and plot values of the variables at fixed points in space against time. As an example, we show in Figure 1 the evolution of the $u$ variable in the high Burger regime at two different spatial points when the mean flow $U_{c}=0.5 \mathrm{~m} \mathrm{~s}^{-1}$. We see that the variable has a fast oscillation, which we calculate to have a period of approximately $300 \mathrm{~s}$. A similar oscillation is seen in the $\phi$ field. Analysis of the model equations shows that this arises from fast-moving gravity waves travelling in the positive $x$-direction at a speed of $20 \mathrm{~m} \mathrm{~s}^{-1}$ relative to the mean flow, which take a time of

$$
\frac{l}{\left(\sqrt{g D}+U_{\mathrm{c}}\right)} \approx 300 \mathrm{~s}
$$

to cover the whole domain, where $D$ is the height of the surface at rest. Since the oscillation is a product of the periodicity of this particular problem, we choose to filter the signal by choosing a time interval of approximately $300 \mathrm{~s}$. For the low Burger regime, the gravity wave speed is much slower relative to the mean flow at $1 \mathrm{~m} \mathrm{~s}^{-1}$ and so no fast oscillation is seen in plots of the different variables against time. Hence we are free to choose the time-differencing interval in the low Burger regime to be also approximately $300 \mathrm{~s}$, without worrying that the statistics will be affected by a dominant signal. Further evidence of the appropriateness of the time intervals chosen is obtained by verifying that the correlations in time between the difference fields decay in time. Details of this analysis can be found in Katz (2007, Appendix A). We now examine the correlations between the control variables in the different dynamical regimes.

\subsection{Correlations in high Burger regime}

We begin by considering the correlations between control variables in a high Burger regime, where we expect the
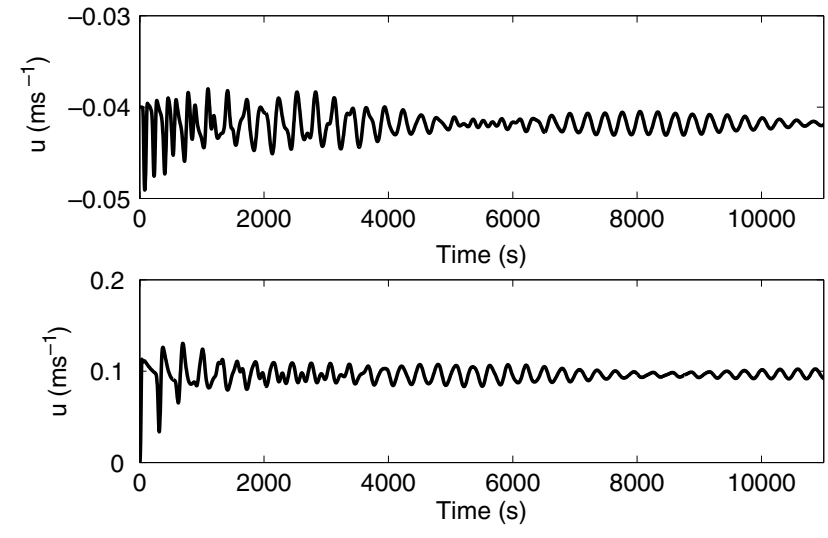

Figure 1. Values of $u$ at spatial points $i=125$ (top) and $i=250$ (bottom), plotted against time for $B u=4.0$.

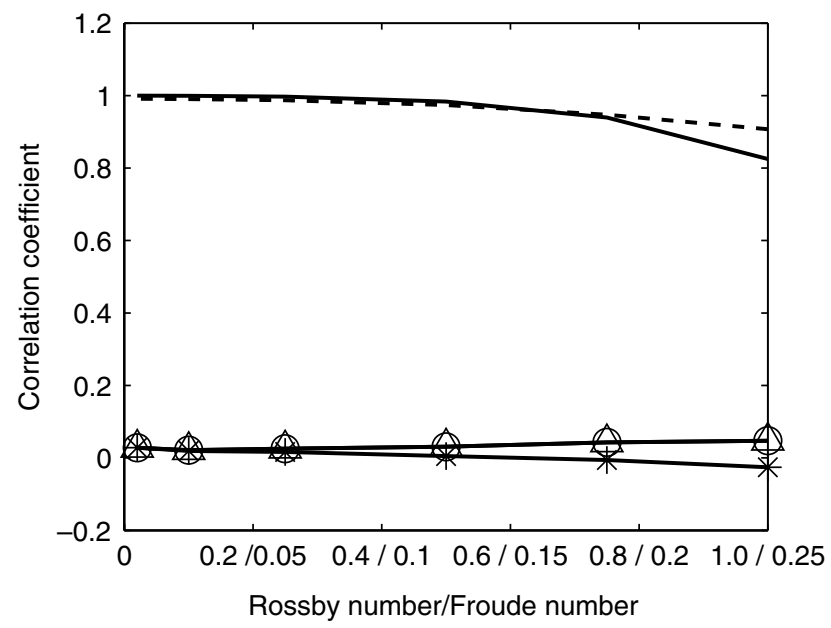

Figure 2. Plot of correlation coefficient against Rossby number for the high Burger number regime. The different lines show the correlations between the full model fields (solid line), between the model increments (dashed line), between vorticity-based control variables $(*)$, and between PV-based variables using the full linearized PV (०) and using the approximate PV $(\triangle)$.

vorticity-based and PV-based transforms to exhibit similar behaviour. The variation in the input values of $U_{c}$ leads to a variation of the Froude number from 0.005 to 0.25 . The time differencing interval is chosen to be

$$
\frac{l}{\left(\sqrt{g D}+U_{c}\right)}=277.5 \mathrm{~s}
$$

where we assume an approximate average mean flow of $U_{\mathrm{c}}=2.5 \mathrm{~m} \mathrm{~s}^{-1}$. We calculate the correlations between full model fields $(\psi, h+\widetilde{H})$, model field increments $\left(\psi^{\prime}, h^{\prime}\right)$, vorticity-based control variables $\left(\psi^{\prime}, h_{\text {res }}^{\prime}\right)$ and PVbased control variables $\left(\psi_{\mathrm{b}}^{\prime}, h_{\mathrm{u}}^{\prime}\right)$. For the PV-based control variables, calculations are made using both the full linearized PV and the approximation (38). The variation of the different correlations with Rossby number is shown in Figure 2.

The first thing we notice is that there is a strong correlation between model variables in both the full fields $(\psi, h+H)$ and the increment fields $\left(\psi^{\prime}, h^{\prime}\right)$, which decreases with Rossby number. This indicates a high degree of balance in the fields. Since the balance approximation (19) is valid in the asymptotic limit of small Rossby number, we see an increase in the correlation as the Rossby number decreases and the flow becomes more balanced. This result helps to validate our choice of statistical method, since this behaviour is exactly as we would expect. 


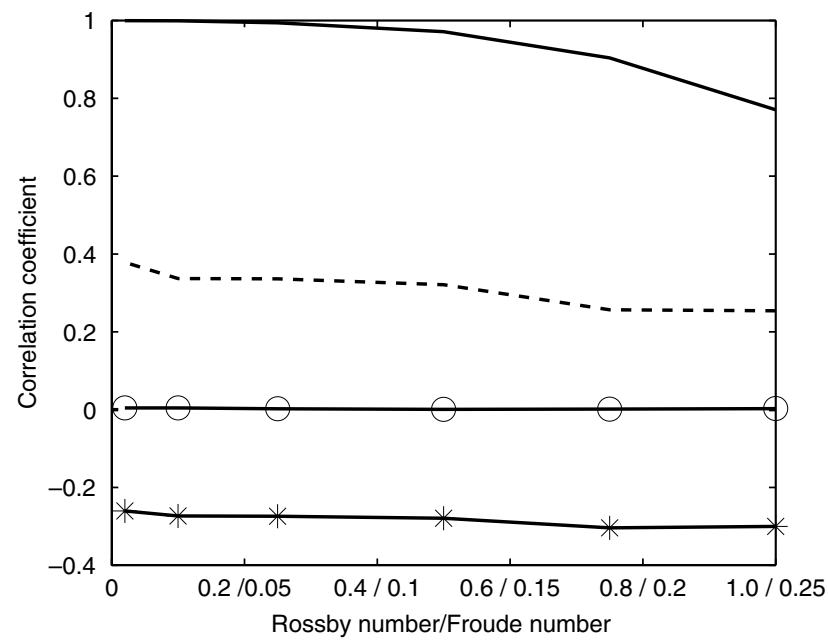

Figure 3. Plot of correlation coefficient against Rossby number for the high Burger regime using a time-differencing interval of $100 \mathrm{~s}$. The lines are labelled as in Figure 2, but the experiment using the approximate PV is not shown.

The correlation between the vorticity-based variables $\left(\psi^{\prime}, h_{\text {res }}^{\prime}\right)$ and between the PV-based variables $\left(\psi_{\mathrm{b}}^{\prime}, h_{\mathrm{u}}^{\prime}\right)$ is much less than the correlation between the model field increments $\left(\psi^{\prime}, h^{\prime}\right)$, with the largest correlations being of order $10^{-2}$. We see that in this high Burger regime both control variable transforms have been successful in removing the strong correlation between model variables. For all values of the Rossby number, the correlations obtained from the two different control variable transforms are very similar. This is to be expected, since in a high Burger regime the balanced variable is approximated well by the vorticity. When the approximation (38) is used in the PVtransform, the correlation between the control variables is almost exactly the same as when we use the full PV variables. The approximation therefore seems to have little effect on the correlation between the PV-based control variables in this case.

To demonstrate that our choice of time interval is appropriate to the high Burger regime, and that the method used to generate the statistics is sensitive to any dominant signal in the data, we compare these results with those obtained when we use a different time interval Figure 3 shows the correlation coefficients using an interval of $100 \mathrm{~s}$, which does not filter the gravity wave signal. These correlations do not correspond with the balances we understand to be present. There is very little balanced correlation in the increments $\psi^{\prime}$ and $h^{\prime}$. However the full model fields $\psi$ and $h+H$ are highly correlated. As the Rossby number is decreased, the balance approximation becomes more accurate and therefore $\psi$ and $h+H$ become increasingly correlated. The same behaviour should also be apparent in the increment fields. However, this is not observed in the correlations. This is because the gravity wave signal is dominating the correlations. A consequence of the lack of correlation between $\psi^{\prime}$ and $h^{\prime}$ in the data is that an unduly strong negative correlation between the vorticitybased variables, $\psi^{\prime}$ and $h_{\text {res }}^{\prime}$, is produced, since from (25) and (26) we have

$$
h_{\mathrm{res}}^{\prime}=h^{\prime}-\frac{f}{g} \psi^{\prime} .
$$

Hence, if the dominant signal of the data is not filtered, then this method for generating the statistics is unable to extract the true correlations which are known to exist.

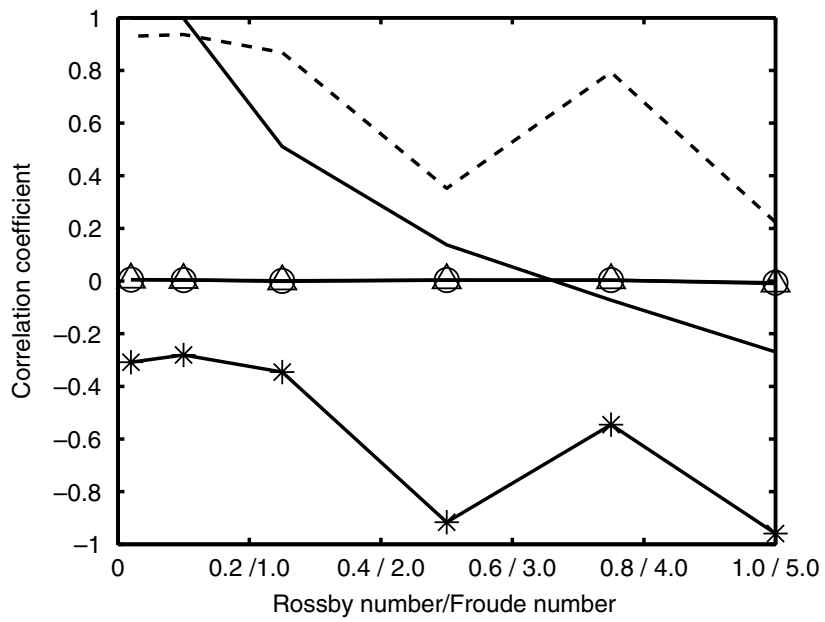

Figure 4. As Figure 2, but for the low Burger regime.

\subsection{Correlations in low Burger regime}

We now consider the correlations between control variables in a low Burger regime, with $B u=0.2$. In this regime the stream function is not completely balanced and we expect the vorticity-based transform to be less effective at removing correlations between variables. The correlations between control variables for this regime are shown in Figure 4. We note that here the Rossby number has the same range as in the high Burger regime, but the Froude number is larger, with the largest value now being 5.0. Here the Froude number is larger than the Rossby number and the flow is dominated by the effects of rotation.

If we examine first the correlations between the full model fields, we note that in this regime, as for the high Burger regime, there is a strong balanced correlation between the full fields for small Rossby number. For Rossby number close to 1.0 , we are not in a low Rossby number regime and we see that for this case the flow is unbalanced. This is observed in the small correlation of $\psi$ and $h+H$. However, a positive correlation remains in the increment fields $\left(\psi^{\prime}, h^{\prime}\right)$. This is attributed to an unbalanced stationary wave in the full model fields that is tied to the orography, which is not seen in the increment fields but does have a signal in the full field correlations. Such a wave would be expected to form when the Froude number increases above 1, leading to a loss of balance in the full fields. This explains the sudden fall in the correlation between $\psi$ and $h+H$ at this value of the Froude number.

The PV-based control variables show a correlation that is close to zero over the whole range of the Rossby number. As in the high Burger regime, this transform is also successful at removing the correlations in a low Burger regime. The vorticity-based control variables on the other hand show a large negative correlation for all values of the Rossby number. It is clear that in the low Burger regime these variables remain highly correlated and so are not a valid choice of control variables for a data assimilation system that assumes the variables are uncorrelated. In this case, the balance is dominated by the mass variables and is not well captured by the vorticity field. Again we find that when the approximation (38) is used in the PV-based transform, the correlations between the control variables are almost identical to those of the unapproximated transform.

\section{Spatial auto-correlations of control variables}

We can gain further insight into the way the control variables decompose the balanced and unbalanced parts of the flow by calculating the spatial auto-correlations for the 
different control variables. These auto-correlations provide information on the inherent length-scale of each variable. This length-scale can be compared to the Rossby radius of deformation $L_{\mathrm{r}}$, defined by (7), and used to assess the degree to which each variable is representing the balanced and unbalanced dynamics of the problem. We expect balanced variables to have a horizontal length-scale $L \approx L_{\mathrm{r}}$, whereas unbalanced variables may have horizontal scales smaller than this, determined by the size of the orography and the value of the mean wind field.

As in section 5, we generate the correlations from a set of 100 time differences, each with 500 grid points. For each control variable, we calculate the correlation between the field at a spatial point $i$ and the field at a spatial point $i+j$ at the same time level for $j=-N / 2, \ldots, N / 2-1$, where $N$ is the number of grid points. The spatial correlation pattern, or correlation function, can then be found by plotting these correlations as a function of the separation distance $j$. In order to compare the auto-correlations produced by the two different parameter transforms, we require a regime in which both balanced and unbalanced motions are present and in which the gravity wave speed is greater than the advective velocity (that is $\mathrm{Fr}<1.0$ ). We use the results of section 5.2 to identify regions in parameter space where there are strong correlations between the model variable increment fields $\left(\psi^{\prime}, h^{\prime}\right)$ and also full fields $(\psi, h+\widetilde{H})$, which indicate that there is a high degree of balance in the flow, without it being totally balanced. For the results of the high Burger experiment shown in Figure 2, we see that the flow is highly balanced for $R o=0.25$, and so we choose the mean flow to be $U_{\mathrm{c}}=1.25 \mathrm{~m} \mathrm{~s}^{-1}$. This gives a Froude number of $\mathrm{Fr}=0.0625$. For the low Burger regime, shown in Figure 4, it is necessary to choose a smaller mean velocity to ensure that the Froude number remains less than 1. Hence we choose $U_{\mathrm{c}}=0.75 \mathrm{~m} \mathrm{~s}^{-1}$, which implies that $R o=0.15$ and $F r=0.75$. By avoiding regimes where the Froude number is greater than 1, we ensure that the fields are not strongly unbalanced.

We consider first the spatial correlations in a high Burger regime. For this experiment $L_{\mathrm{r}}$, calculated from (7), is $2000 \mathrm{~m}$, which corresponds to 160 grid points. In Figure 5 we plot the correlation functions corresponding to a point in the centre of the domain for the vorticity-based and PV-based variables. We see that the correlation scale is largest for the balanced variables $\psi^{\prime}$ and $\psi_{\mathrm{b}}^{\prime}$ and is of order approximately $L_{\mathrm{r}}$. The correlation scales for the unbalanced variables $h_{\text {res }}^{\prime}$ and $h_{\mathrm{u}}^{\prime}$ are less than the Rossby deformation radius. These results indicate that both the vorticity-based and PV-based variables are capturing the balanced and unbalanced flows well in this regime.

For the low Burger regime, the Rossby radius is much smaller and is equal to approximately $100 \mathrm{~m}$, or 8 grid points. In Figure 6 we plot the correlation functions calculated in this regime. We would expect that correlations for the unbalanced height variable to be on a scale of less than $100 \mathrm{~m}$ if it accurately represents the unbalanced motion. We see that this is the case for the PV-based variable $h_{\mathrm{u}}^{\prime}$ but not the vorticity-based variable $h_{\text {res }}^{\prime}$. In this regime the balanced part of the flow is dominated by the height and the vorticity-based variables do not account for this. Hence we see correlation structures for $h_{\text {res }}^{\prime}$ on too large a scale. We can therefore conclude that the PV-based variables are accurately capturing the correct balances in the low Burger regime while the vorticity-based variables are not.

\section{Conclusions}

The ability of the parameter transform to split the flow into uncorrelated parts is an important assumption of many operational variational data assimilation systems. A decomposition of the variables based on PV has previously (a)
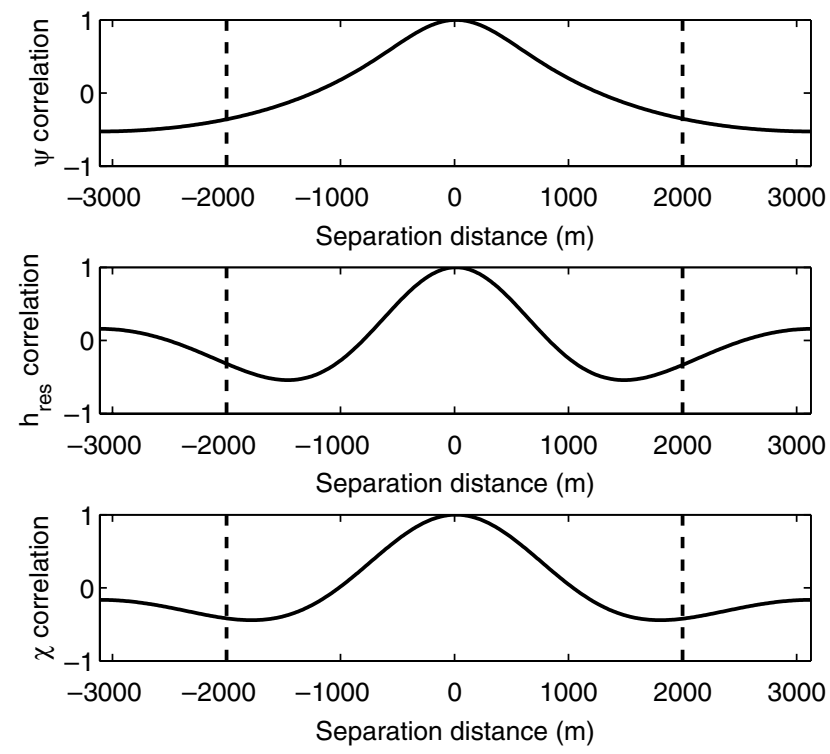

(b)
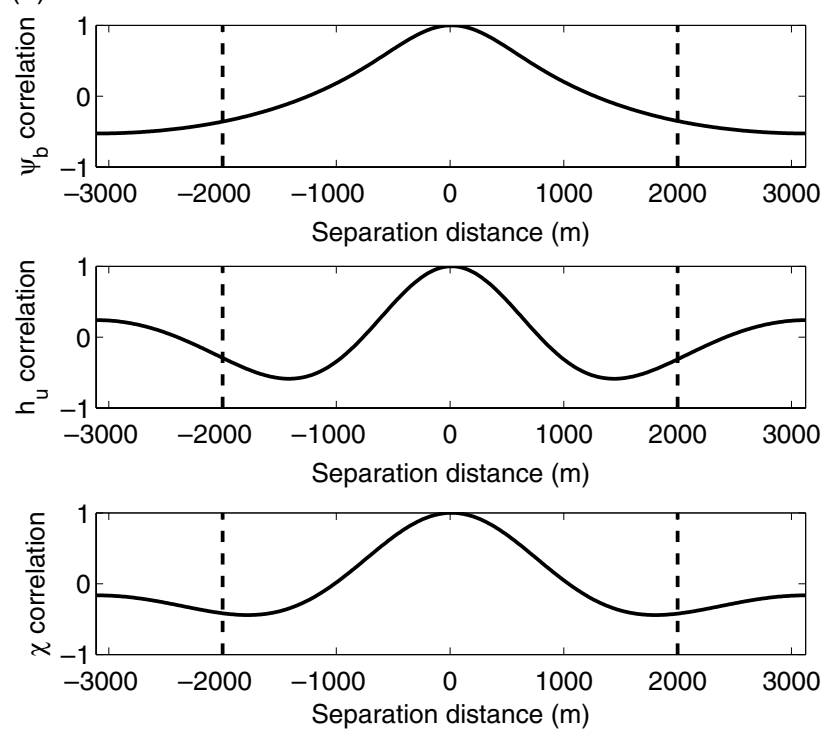

Figure 5. Correlation functions in the high Burger regime for (a) vorticitybased variables $\psi$ (top), $h_{\text {res }}$ (middle) and $\chi$ (bottom) and for (b) PV-based variables $\psi_{\mathrm{b}}$ (top), $h_{\mathrm{u}}$ (middle) and $\chi$ (bottom). The Rossby radius is marked with a dashed line.

been proposed in the literature as being more valid than the commonly used vorticity-based decomposition. In this study we have demonstrated that the approximate transform based on vorticity does effectively remove correlations in a high Burger regime, but that the control variables are highly correlated in a low Burger regime. This is explained by the fact that the balanced part of the flow in the low Burger regime is best represented by the mass variables rather than the rotational part of the wind. Thus the stream function, which is used as the balanced variable in this transform, is unable to represent the balanced flow correctly. On the other hand, the PV-based variables are able to decouple the flow successfully for both the high and low Burger regimes. As expected from previous theoretical studies, the splitting of the stream function into balanced and unbalanced components in this transform allows a better representation of balance over a wider range of dynamical regimes. A key assumption of this method is that the flow can be separated into a balanced component, dominated by the PV, and an unbalanced component, dominated by gravity waves. Although this assumption may not be true 
(a)
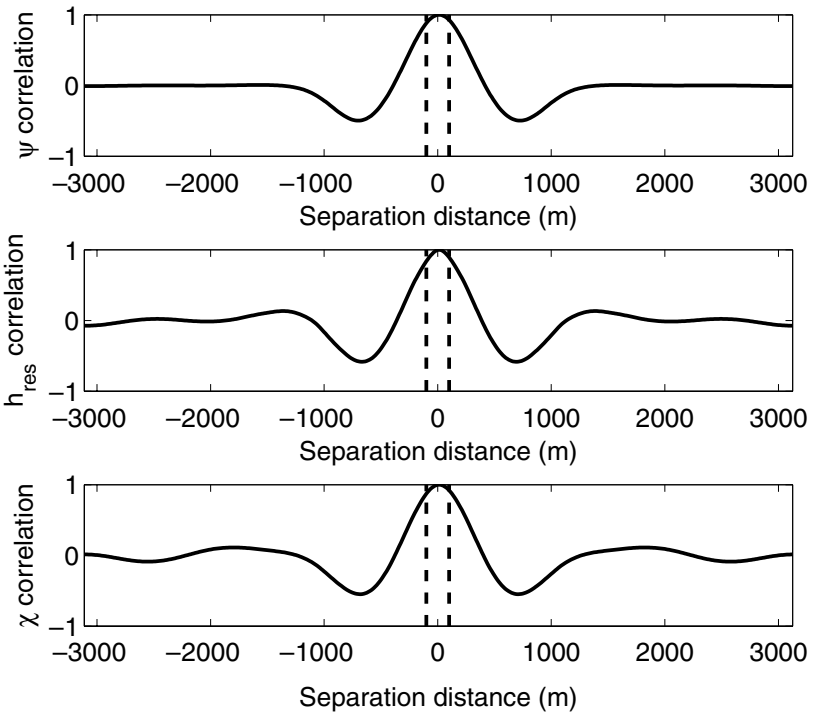

(b)
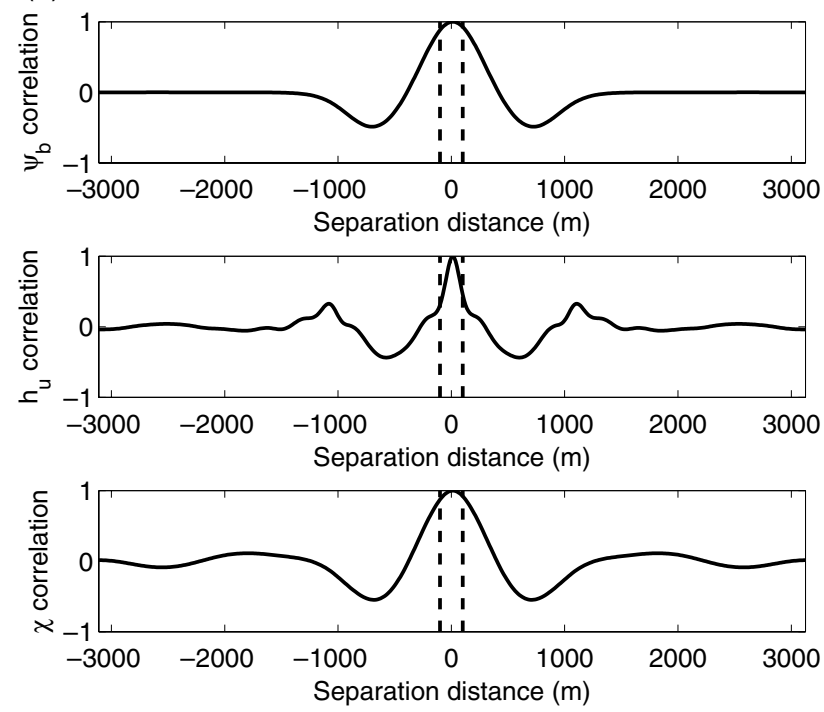

Figure 6. As Figure 5, but for the low Burger regime.

everywhere in the atmosphere, within a real assimilation system it is necessary to make assumptions that are valid most of the time and for most of the atmosphere. This work indicates that the assumptions made when using the PV-based transformation are more valid in this respect than those made using the vorticity-based transfromation.

We note that, although we have been concerned here with the application of the control variable transform in a standard variational assimilation setting, these results may also have implications for correlations obtained by ensemble-based techniques. Mitchell et al. (2002) demonstrated that the localization needed in an ensemble Kalman filter could destroy balances which are present. More recently Kepert (2009) has shown in a shallow-water model that the balance can be better preserved by performing localization in the stream function and velocity potential variables rather than the wind components. Thus, even within ensemble methods, the correct assumption as to which variables are correlated is likely to be essential in order to obtain a good performance of the assimilation.

Despite the evident advantage in using the PVbased transform, issues still remain with regard to its implementation in data assimilation systems. As noted by Bannister and Cullen (2007), the use of a transform based on $\mathrm{PV}$ in a full numerical weather prediction system requires the iterative solution of a three-dimensional elliptic equation, which is not well conditioned. Cullen (2003) commented that this poor conditioning of the transform could lead to slower convergence of the assimilation, even though the assumption of uncorrelated errors is more realistic. Hence further preconditioning techniques need to be developed in order to implement the transform efficiently. Nevertheless the results of this study indicate that there is much advantage to be gained from using a parameter transform based on PV and so the effort towards an efficient numerical implementation should be worthwhile.

\section{Acknowledgements}

This work was supported by the Engineering and Physical Sciences Research Council and the Met Office through a CASE studentship, and in part by the Natural Environment Research Council.

\section{References}

Bannister RN. 2008a. A review of forecast-error covariance statistics in atmospheric variational data assimilation. I: Characteristics and measurements of forecast-error covariances. Q. J. R. Meteorol. Soc. 134: $1951-1970$

Bannister RN. 2008b. A review of forecast-error covariance statistics in atmospheric variational data assimilation. II: Modelling the forecasterror covariance statistics. Q. J. R. Meteorol. Soc. 134: 1971-1996.

Bannister RN, Cullen MJP. 2007. 'A regime-dependent balanced control variable based on potential vorticity'. In Proceedings of Workshop on Flow-dependent Aspects of Data Assimilation, 11-13 June 2007. ECMWF: Reading, UK. 101-112.

Bannister RN, Katz D, Cullen MJP, Lawless AS, Nichols NK. 2008. Modelling of forecast errors in geophysical fluid flows. Int. J. Numer. Methods Fluids 56: 1147-1153.

Barker DM, Huang W, Guo Y-R, Bourgeois AJ, Xiao QN. 2004. A three-dimensional variational data assimilation system for MM5: Implementation and initial results. Mon. Weather Rev. 132: 897-914.

Courtier P, Thépaut J-N, Hollingsworth A. 1994. A strategy for operational implementation of $4 \mathrm{D}$-Var, using an incremental approach. Q. J. R. Meteorol. Soc. 120: 1367-1387.

Cullen MJP. 2003. Four-dimensional variational data assimilation: A new formulation of the background-error covariance matrix based on a potential vorticity representation. Q. J. R. Meteorol. Soc. 129: 2777-2796.

Derber J, Bouttier F. 1999. Formulation of the background-error covariances in the ECMWF global data assimilation system. Tellus 51A: 195-221.

Dritschel DG, Polavani LM, Mohebalhojeh AR. 1999. The contouradvective semi-Lagrangian algorithm for the shallow-water equations. Mon. Weather Rev. 127: 1551-1565.

Fischer C, Montmerle T, Berre L, Auger L, Stefanescu SE. 2005. An overview of the variational assimilation in the ALADIN/France numerical weather prediction system. Q. J. R. Meteorol. Soc. 131: 3477-3492.

Fisher M. 2003. 'Background-error covariance modelling'. In Proceedings of Seminar on Recent Developments in Data Assimilation for Atmosphere and Ocean, 8-12 Sept 2003. ECMWF: Reading, UK. 45-63.

Fletcher SJ. 2004. 'High-order balance conditions using Hamiltonian dynamics for numerical weather prediction'. PhD thesis, Department of Mathematics, University of Reading, UK.

Gauthier P, Charette C, Fillion L, Koclas P, Laroche S. 1999. Implementation of a $3 \mathrm{D}$ variational data assimilation system at the Canadian Meteorological Centre. Part 1: The global analysis. Atmos.-Ocean 37: 103-156.

Hoskins BJ, McIntyre ME, Robertson AW. 1985. On the use and significance of isentropic potential vorticity maps. Q. J. R. Meteorol. Soc. 111: 877-946.

Jackson DR, Keil M, Devenish BJ. 2008. Use of Canadian Quick covariances in the Met Office data assimilation scheme. Q. J. R. Meteorol. Soc. 134: 1567-1582.

Katz D. 2007. 'The appplication of PV-based control variable transformations in variational data assimilation'. $\mathrm{PhD}$ thesis, Department of Mathematics, University of Reading, UK.

Kepert JD. 2009. Covariance localisation and balance in an Ensemble Kalman Filter. Q. J. R. Meteorol. Soc. 135: 1157-1176. 
Lawless AS, Nichols NK, Ballard SP. 2003. A comparison of two methods for developing the linearization of a shallow-water model. Q. J. R. Meteorol. Soc. 129: 1237-1254.

Lawless AS, Gratton S, Nichols NK. 2005. An investigation of incremental 4D-Var using non-tangent linear models. Q. J. R. Meteorol. Soc. 131: 459-476.

Lorenc AC. 2003. Modelling of error covariances by 4D-Var data assimilation. Q. J. R. Meteorol. Soc. 129: 3167-3182.

Lorenc AC, Ballard SP, Bell RS, Ingleby NB, Andrews PLF, Barker DM, Bray JR, Clayton AM, Dalby T, Li D, Payne TJ, Saunders FW. 2000. The Met Office global 3-dimensional variational data assimilation scheme. Q. J. R. Meteorol. Soc. 126: 2991-3012.

Mitchell HL, Houtekamer PL, Pellerin G. 2002. Ensemble size, balance and model-error representation in an ensemble Kalman filter. Mon. Weather Rev. 130: 2791-2808.
Mohebalhojeh AR, Dritschel DG. 2004. Contour-advective semiLagrangian algorithms for many-layer primitive-equation models. Q. J. R. Meteorol. Soc. 130: 347-364.

Parrish D, Derber JC. 1992. The National Meteorological Center's Spectral Statistical-Interpolation Analysis System. Mon. Weather Rev. 120: $1747-1763$.

Pedlosky J. 1987. Geophysical Fluid Dynamics. Springer-Verlag: New York, NY.

Polavarapu S, Ren S, Rochon Y, Sankey D, Ek N, Koshyk J, Tarasick D. 2005. Data assimilation with the Canadian Middle Atmosphere Model. Atmos.-Ocean 43: 77-100.

Wlasak M, Nichols NK, Roulstone I. 2006. Use of potential vorticity for incremental data assimilation. Q. J. R. Meteorol. Soc. 132: 2867-2886.

Zupanski M, Zupanski D, Vukicevic T, Els K, Vonder Haar T. 2005. CIRA/CSU four-dimensional variational data assimilation system. Mon. Weather Rev. 133: 829-843. 